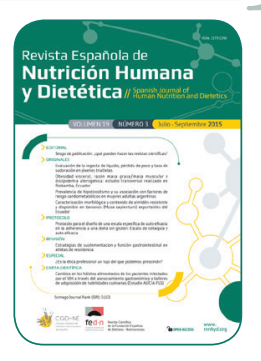

\title{
EDITORIAL
}

\section{Sesgo de publicación: ¿qué pueden hacer las revistas científicas?}

\author{
Eduard Baladia $^{a, b, *}$, Rodrigo Martínez-Rodríguez ${ }^{a, b}$ \\ a Centro de Análisis de la Evidencia Científica de la Fundación Española de Dietistas-Nutricionistas (CAEC-FEDN), España. \\ b Revista Española de Nutrición Humana y Dietética, España. \\ *baladia@ebaladia.es
}

Recibido el 29 de septiembre de 2015; aceptado el 30 de septiembre de 2015.

\section{CITA}

Baladia E, Martínez-Rodríguez R. Sesgo de publicación: ¿qué pueden hacer las revistas científicas?. Rev Esp Nutr Hum Diet. 2015; 19(3): 130 - 131. DOI: 10.14306/renhyd.19.3.186

El sesgo de publicación es el fenómeno que ocurre cuando los datos que aparecen en la literatura publicada son sistemáticamente no representativos del conjunto de datos recopilados en los estudios realizados ${ }^{1}$. Existen evidencias fuertes de que los resultados con datos estadísticamente significativos y positivos (a favor de la hipótesis), tienen más posibilidades de ser publicados ${ }^{1}$.

En consecuencia, la no publicación de datos con efectos nulos, negativos o estadísticamente no significativos ${ }^{2}$, puede estar conduciendo a la sobreestimación de los efectos de algunos tratamientos en los correspondientes metaanálisis ${ }^{3}$ y, de este modo, afectando al juicio y práctica clínica basada en la evidencia.

Las razones y causas de la existencia del sesgo de publicación han sido ampliamente investigadas y tienen varios orígenes, algunos de las cuales son responsabilidad de los autores/as y otras del sistema editorial y de las revistas científicas.

El sesgo de publicación se ha asociado, clásicamente, a la presencia del patrocinio de la industria, cuyos estudios suelen producir más proporción de resultados positivos que aquellos patrocinados por otro tipo de fuentes de financiación ${ }^{4}$ y menos reporte de efectos adversos (sesgo de información) ${ }^{5}$. Asimismo, los propios investigadores también tienden a retrasar la publicación de datos menos espectaculares ${ }^{6}$, a veces por razones económicas ${ }^{7}$, convirtiéndose en datos de cajón o sobremesa que quizás nunca lleguen a ser publicados. Incluso entre autores Cochrane, los cuales están especialmente sensibilizados con el sesgo de publicación, se estima que dos terceras partes de sus datos no serán publicados en revistas científicas incluso después de 5 años de su presentación en su congreso, lo que subraya la necesidad de buscar en resúmenes de conferencias si se quieren obtener todas las pruebas existentes $^{8}$.

Pese a lo dicho, también existen factores asociados al proceso editorial o a intereses de las revistas científicas que no pueden ni deben ser menospreciados. Pese a que en un metaanálisis sobre el alcance del sesgo de publicación, en 20099, no se observó la influencia de la direccionalidad de los datos en la decisión editorial, el carácter prospectivo de los estudios incluidos podría haber modificado la conducta de decisión editorial, al conocer el Comité Editorial que 
su revista estaba siendo investigada. Asimismo, las revistas suelen obtener mayor puntuación de factor de impacto mediante la publicación de estudios financiados por la industria debido a que este tipo de estudios serán más citados en otras revistas (seguramente incentivado por la propia industria) ${ }^{10}$ y también suelen tener más ingresos con estudios financiados por la industria a través de las ventas de reimpresiones y separatas (suelen querer más cantidad para presentar en congresos y foros científicos) ${ }^{11}$. Pese a lo dicho, un estudio retrospectivo realizado en ocho revistas médicas, observó que ninguno de dichos aspectos había influenciado (aparentemente) en la decisión editorial, indicando que también existe un creciente interés por parte de las revistas en realizar un manejo ético y que minimice el sesgo de publicación ${ }^{12}$.

Para minimizar el sesgo de publicación se han delineado varias opciones que incluyen desde la aplicación de modelos estadísticos en la evaluación de las evidencias, hasta la creación de estrategias de información y comunicación de su importancia o la elaboración de políticas de transparencia sobre el desarrollo y la publicación de investigación $n^{7,13}$. En los últimos años, quizás gracias a la campaña \#AlITrials (http://www.alltrials.net/), el registro y publicación de protocolos ha adquirido un creciente interés y relevancia en la intención de minimizar el sesgo de publicación y evitar la modificación de protocolos una vez iniciada la investigación ${ }^{14}$.

La Revista Española de Nutrición Humana y Dietética puede decir desde 2013 que no alberga publicidad alguna (por lo que no presenta posibles conflictos de interés en la publicación de datos contrarios a los intereses de los financiadores de la Revista), que es Open Access sin cargo a los autores/as (no se beneficia de la publicación de artículos y facilita la publicación de datos de autores que no disponen de financiación), que no tiene ingresos por la reimpresión y edición de separatas (porque no las realiza), que obliga a sus autores a emitir una declaración de conflictos de interés personal o financiero y que desde sus inicios se ha comprometido a publicar todos aquellos datos que deriven de estudios con metodología adecuada independientemente de la direccionalidad o significancia de sus resultados.

La Revista acaba de abrir una nueva sección de publicación de protocolos y dará prioridad de publicación a aquellos estudios que gocen de registro del protocolo en cualquiera de las bases de datos disponibles (a nivel mundial, europeo o nacional). A partir de hoy, también podrá decir que se une a la lucha contra el sesgo de publicación.

\section{BIBLIOGRAFÍA}

1. Dwan K, Gamble C, Williamson PR, Kirkham J], Reporting Bias Group. Systematic review of the empirical evidence of study publication bias and outcome reporting bias - an updated review. PloS One. 2013; 8(7): e66844.

2. Hopewell S, Loudon K, Clarke MJ, Oxman AD, Dickersin K. Publication bias in clinical trials due to statistical significance or direction of trial results. Cochrane Database Syst Rev. 2009; (1): MR000006.

3. Onishi A, Furukawa TA. Publication bias is underreported in systematic reviews published in high-impact-factor journals: metaepidemiologic study. J Clin Epidemiol. 2014; 67(12): 1320-6.

4. Lundh A, Sismondo S, Lexchin J, Busuioc OA, Bero L. Industry sponsorship and research outcome. Cochrane Database Syst Rev. 2012; 12: MR000033.

5. Als-Nielsen B, Chen W, Gluud C, Kjaergard LL. Association of funding and conclusions in randomized drug trials: a reflection of treatment effect or adverse events? JAMA. 2003; 290(7): 921-8.

6. Malički M, Marušić A. Is there a solution to publication bias? Researchers call for changes in dissemination of clinical research results. J Clin Epidemiol. 2014; 67(10): 1103-10.

7. Kien C, Nußbaumer B, Thaler KJ, Griebler U, Van Noord MG, Wagner $P$, et al. Barriers to and facilitators of interventions to counter publication bias: thematic analysis of scholarly articles and stakeholder interviews. BMC Health Serv Res. 2014; 14: 551.

8. Chapman S, Eisinga A, Hopewell S, Clarke M. Two-thirds of methodological research remained unpublished after presentation at Cochrane Colloquia: an empirical analysis. ] Clin Epidemiol. 2012; 65(5): 493-6.

9. Song F, Parekh-Bhurke S, Hooper L, Loke YK, Ryder J], Sutton $A]$, et al. Extent of publication bias in different categories of research cohorts: a meta-analysis of empirical studies. BMC Med Res Methodol. 2009; 9: 79.

10. Lundh A, Barbateskovic M, Hróbjartsson A, Gøtzsche PC. Conflicts of interest at medical journals: the influence of industry-supported randomised trials on journal impact factors and revenue - cohort study. PLoS Med. 2010; 7(10): e1000354.

11. Handel AE, Patel SV, Pakpoor J, Ebers GC, Goldacre B, Ramagopalan SV. High reprint orders in medical journals and pharmaceutical industry funding: case-control study. BM]. 2012; 344: e4212.

12. van Lent $M$, Overbeke J, Out $\mathrm{HJ}$. Role of Editorial and Peer Review Processes in Publication Bias: Analysis of Drug Trials Submitted to Eight Medical Journals. PLoS ONE [Internet]. 12 de agosto de 2014 [citado 30 de septiembre de 2015]; 9(8). Recuperado a partir de: http://www.ncbi.nIm.nih.gov/pmc/ articles/PMC4130599/

13. Thaler K, Kien C, Nussbaumer B, Van Noord MG, Griebler U, Klerings $I$, et al. Inadequate use and regulation of interventions against publication bias decreases their effectiveness: a systematic review. J Clin Epidemiol. 2015; 68(7): 792-802.

14. Rezaeian M. Reducing publication bias in biomedical research: reviewing and registering protocol with a suitable journal. ] Clin Epidemiol. 2015; in Press. 